Check for updates

Cite this: Phys. Chem. Chem. Phys., 2017, 19, 19904

Received 12th June 2017, Accepted 9th July 2017

DOI: $10.1039 / c 7 c p 03922 k$

rsc.li/pccp

\section{An axis-specific rotational rainbow in the direct scatter of formaldehyde from Au(111) and its influence on trapping probability $\dagger$}

\author{
G. Barratt Park, (D)*ab Bastian C. Krüger, (D) a Sven Meyer, ${ }^{a}$ Alexander Kandratsenka, (iD b \\ Alec M. Wodtke iD ab and Tim Schäfer iD a
}

\begin{abstract}
The conversion of translational to rotational motion often plays a major role in the trapping of small molecules at surfaces, a crucial first step for a wide variety chemical processes that occur at gas-surface interfaces. However, to date most quantum-state resolved surface scattering experiments have been performed on diatomic molecules, and little detailed information is available about how the structure of nonlinear polyatomic molecules influences the mechanisms for energy exchange with surfaces. In the current work, we employ a new rotationally resolved $1+1^{\prime}$ resonance-enhanced multiphoton ionization (REMPI) scheme to measure the rotational distribution in formaldehyde molecules directly scattered from the $\mathrm{Au}(111)$ surface at incidence kinetic energies in the range $0.3-1.2 \mathrm{eV}$. The results indicate a pronounced propensity to excite a-axis rotation (twirling) rather than $b$ - or $c$-axis rotation (tumbling or cartwheeling), and are consistent with a rotational rainbow scattering model. Classical trajectory calculations suggest that the effect arises-to zeroth order-from the three-dimensional shape of the molecule (steric effects). Analysis suggests that the high degree of rotational excitation has a substantial influence on the trapping probability of formaldehyde at incidence translational energies above $0.5 \mathrm{eV}$.
\end{abstract}

\section{Introduction}

The interconversion of translational and rotational energy in molecule-surface collisions can play a key role in the mechanism for adsorption, ${ }^{1-5}$ which is the crucial first step for a large number of chemical processes that take place at gas-surface interfaces. ${ }^{6,7}$ This has motivated a large number of quantumstate resolved measurements of rotational distributions in molecules scattered or desorbed from surfaces, which provide detailed information not only on the mechanism for trapping/ desorption, ${ }^{2,8-10}$ but also on orientation dependence of moleculesurface interactions (steric effects). ${ }^{11-16}$ The rotational distributions obtained from direct (i.e. single-collision-event) molecular scattering experiments are often non-thermal, and striking features can arise from so-called "rotational rainbow" effects, which occur when there is a singularity in the classical rotational

\footnotetext{
${ }^{a}$ University of Goettingen, Institute for Physical Chemistry, Tammannstr. 6, 37077 Goettingen, Germany. E-mail: barratt.park@mpibpc.mpg.de

${ }^{b}$ Max Planck Institute for Biophysical Chemistry, Göttingen, Am Fassberg 11, 37077 Goettingen, Germany

$\dagger$ Electronic supplementary information (ESI) available: Backing gas mixtures, method for fitting the spectra, rotational distributions from the trappingdesorption channel, tabulation of parameters for the direct scatter rotational distributions, and rotational state dependence of the scattered beam velocity. See DOI: $10.1039 / \mathrm{c} 7 \mathrm{cp} 03922 \mathrm{k}$
}

energy distribution due to an extremum in $J(\gamma)$, (where $J$ is the rotational angular momentum in the scattering product and $\gamma$ is the orientation of the incoming molecule). ${ }^{17}$ Such effects are analogous to those caused by extrema in the classically achievable scattering angle of light from water droplets-hence the name. The consequence of an extremum in $J(\gamma)$ is that there is a range of initial $\gamma$ leading to approximately the same $J$, such that population in the scattering products accumulates at that $J$. Quantum effects lead to broadened maxima, rather than sharp singularities, in the rotational state distributions observed in molecular beam scattering experiments. ${ }^{18}$ Classical and quantum calculations based on model potentials have succeeded in reproducing the key features of the experimentally observed rotational rainbow effects in a variety of systems, displaying diverse behavior. ${ }^{19-25}$

The excitation of molecular degrees of freedom becomes increasingly important in the dissipation of incident kinetic energy during molecule/surface collisions as the number of atoms in the molecule is increased. In fact, rotational excitation has been invoked as a key component of the trapping mechanism in a number of polyatomic molecule-surface systems. ${ }^{4,5}$ However, to date, almost all experiments that probe quantumstate resolved rotational excitation at surfaces have been limited to diatomic or linear polyatomic ${ }^{26,27}$ molecules. The degree to which nonlinear polyatomic molecules are rotationally excited 
during collisions with surfaces remains an experimentally unexplored area. We are aware of only two previous surface scattering experiments that have probed the rotational distribution in a scattered nonlinear polyatomic molecule: $\mathrm{CH}_{4}$ from $\mathrm{LiF}(100)^{28}$ and $\mathrm{NH}_{3}$ from $\mathrm{Au}(111) .^{25,29}$ The $\mathrm{CH}_{4}$ study, which was conducted at an incidence energy of $0.075 \mathrm{eV}$ and an incidence angle of $60^{\circ}$, found no significant deviation from a thermal rotational distribution. On the other hand, the $\mathrm{NH}_{3}$ study, which was conducted at an incidence energy of $0.24 \mathrm{eV}$ and an incidence angle of $45^{\circ}$, found a strong propensity for a rotationally cold $\mathrm{NH}_{3}$ beam to scatter elastically into low- $J$ states. A propensity to excite low- $K$ states (tumbling rather than twirling) was also observed. The results were interpreted in terms of a dynamical steering effect, which orients the symmetry axis of the $\mathrm{NH}_{3}$ molecule along the surface normal prior to collision.

In the current work, we report rotationally-resolved molecular beam scattering of formaldehyde from $\mathrm{Au}(111)$. We report an axis-specific rotational rainbow in the direct scattering channel. Unlike in the case of $\mathrm{NH}_{3}$, direct scatter of formaldehyde from $\mathrm{Au}(111)$ leads to a clear propensity for excitation of rotational states with a high degree of rotational energy about the symmetry axis. The results are interpreted with the aid of classical trajectory calculations. To our knowledge, the present work is the first report of rotationally resolved surface scattering of an asymmetric top molecule and the first report of a high- $J$ rotational rainbow in a nonlinear molecule. The work demonstrates the ability to probe the highly structured steric effects that govern the interactions between polyatomic molecules and surfaces, and the results suggest that rotational excitation contributes to the trapping probability of formaldehyde on $\mathrm{Au}(111)$ at high incidence kinetic energies.

\section{Methods}

\section{A. Experimental}

The essential details of the experiment are described in ref. 30 . A pulsed beam of formaldehyde was generated by cracking solid paraformaldehyde ( $97 \%$ purity) in a sample holder inside the nozzle of a home-built pulsed solenoid valve, described in ref. 31. The tip of the nozzle was heated to a temperature of 104-130 ${ }^{\circ} \mathrm{C}$ and the sample holder was maintained at a cracking temperature of $60-80{ }^{\circ} \mathrm{C}$. The molecular expansion is sent through a $2 \mathrm{~mm}$ electroformed skimmer (Ni Model 2, Beam Dynamics, Inc.) located $6 \mathrm{~cm}$ from the nozzle and through a second $2 \mathrm{~mm}$ aperture, located $20 \mathrm{~cm}$ downstream, before entering the ultra-high vacuum (UHV) chamber, where the surface scattering experiments are performed. We calculate the peak density of the incoming beam in the UHV chamber to be $7 \times 10^{11} \mathrm{~cm}^{-3}$, so that the mean free path of scattered molecules traveling through the incoming beam can be conservatively estimated to be $\gtrsim 1 \mathrm{~m}$, and collisions between molecules can be excluded.

The incidence kinetic energy $\left(E_{\mathrm{i}}\right)$ of the incoming beam of formaldehyde was varied over the range 0.1-1.2 eV, by using different backing gas mixtures (see Table SI of the ESI $\dagger$ ).
The stagnation pressure behind the nozzle was also varied over the range 3-12 bar to achieve a 3-15 K rotational temperature $\left(T_{\text {rot }}\right)$ in the incoming molecular beam. Under these conditions, the incoming beams used in this study had no significant population in levels with $K_{a}>1$ or $J>4$.

The incoming beam was scattered at near-normal incidence angle from a $\mathrm{Au}(111)$ crystal (Mateck). Before each experiment, the $\mathrm{Au}(111)$ crystal is cleaned by sputtering with an Ar-ion gun (LK Technologies NGI3000) and annealed for 20 minutes at 900 K. Surface cleanliness is verified by Auger electron spectroscopy (Staib Instruments, Inc. ESA-150). The temperature $\left(T_{\mathrm{s}}\right)$ of the $\mathrm{Au}(111)$ crystal was monitored by a thermocouple (K type), and could be cooled by liquid nitrogen or heated resistively to achieve temperatures in the range 100-1000 K.

The rotational distribution in the vibronic ground state was monitored in the incoming and scattered molecular beam via a $1+1^{\prime}$ REMPI scheme, which we recently reported. ${ }^{32}$ Briefly, the frequency-doubled output of a pulsed dye laser (Sirah Cobra Stretch SL, operating with LDS 698 or 722 dye), pumped at $10 \mathrm{~Hz}$ by a Nd:YAG laser (Continuum Powerlite 8010), was scanned across the rotationally resolved $\tilde{\mathrm{A}}^{1} \mathrm{~A}_{2} \leftarrow \tilde{\mathrm{X}}^{1} \mathrm{~A}_{1}\left(4_{0}^{1}\right)$ transition at $353 \mathrm{~nm}$. (We use the notation $4_{v_{4}^{\prime \prime}}^{v_{4}^{\prime}}$ to denote the number of quanta of out-of-plane wagging vibration $\nu_{4}$ in the upper $\left(v_{4}^{\prime}\right)$ and lower $\left(v_{4}^{\prime \prime}\right)$ electronic state, respectively.) The pulse energy was typically 5-7 $\mathrm{mJ}$ and the beam was collimated to a diameter of $\sim 3 \mathrm{~mm}$. A small portion of the dye laser fundamental was coupled into a high-precision wavemeter (HighFinesse, WS7) for frequency calibration, to an accuracy of $\pm 0.02 \mathrm{~cm}^{-1} \cdot{ }^{31}$ Variation in the laser intensity over the scan was monitored by recording the shot-by-shot pulse energy behind the chamber. After a delay of $20 \mathrm{~ns}$, molecules were ionized from the Ã state by a single vacuum ultraviolet (VUV) photon at $157 \mathrm{~nm}$, obtained from a molecular $\mathrm{F}_{2}$ laser (GAM EX350). The VUV beam (2-8 mJ pulse energy) was counter-propagated relative to the dye laser beam and was focused into the interaction region by a $f=58.1 \mathrm{~cm} \mathrm{CaF}_{2}$ lens. The experiment is operated in a regime where the signal is linear with respect to both laser pulses, and the relative integrated ion intensities are directly proportional to the $\tilde{\mathrm{A}} \leftarrow \tilde{\mathrm{X}}$ absorption cross section to within $\sim 20 \%$ uncertainty. ${ }^{32}$ Molecular cations were collected by a set of ion optics and detected on a 2-stage microchannel plate assembly (Hamamatsu F1552) in chevron configuration.

At $E_{\mathrm{i}}=0.1 \mathrm{eV}$, the scattering is dominated by a trapping/ desorption mechanism in which molecules physisorb to the surface before undergoing thermal desorption. At higher incidence energies $\left(E_{\mathrm{i}}=0.3-1.2 \mathrm{eV}\right)$, we observe distinct channels for trapping/desorption and direct (single-impact) scattering. These channels have been characterized in detail in ref. 30. At incidence energies between $0.33-0.39 \mathrm{eV}$ we probe the scattered molecules at a distance of $9 \mathrm{~mm}$ from the surface, $3 \mathrm{~mm}$ above the incoming beam. This allows us to capture a narrow range of near-specular scattering angles, in which the direct scatter channel dominates. At incidence energies greater than $0.39 \mathrm{eV}$ the direct scatter channel dominates over a wider range of scattering angle. At high $E_{\mathrm{i}}$, the direct scattering channel can 
also be resolved from the trapping/desorption channel and from the incoming beam via ion time-of-flight (TOF) to the detector. $^{30}$ The scattering products in the $E_{\mathrm{i}}=0.48-1.2 \mathrm{eV}$ range were thus monitored $3 \mathrm{~mm}$ from the surface, such that a wide range of scattering angles is probed. No significant variation of rotational distribution was found over the range of distances and angles probed in our experiment.

\section{B. Fitting and simulation}

For comparison with our measured spectra, we calculate the frequency and absorption intensity of each line using a Watson asymmetric top Hamiltonian, with the parameters of ref. 33 for the $\tilde{\mathrm{A}} 4^{1}$ state and the parameters of ref. 34 for the ground state. The lines in the $b$-type $\tilde{\mathrm{A}} \leftarrow \tilde{\mathrm{X}}\left(4_{0}^{1}\right)$ transition are sufficiently dense that few individual rotational lines are fully resolved in the spectra of rotationally hot scattering products, obtained with a dye laser resolution of $\sim 0.1 \mathrm{~cm}^{-1}$. Therefore, a Boltzmann plot analysis based on individual integrated linestrengths is not possible. However, our resolution is sufficient to obtain many partially resolved lineshapes that give rise to sharp features in the spectra, even in the hottest rotational distributions observed. We have made detailed manual feature-by-feature analyses of the rotational structure, and we have supplemented this analysis by fitting the observed spectra to simulated spectra obtained by modeling the rotational distribution. Additional details about the fitting method are given in Section SII of the ESI. $\dagger$

\section{Results and analysis}

\section{A. Trapping/desorption channel: dependence of the rotational distribution on $T_{\mathrm{s}}$}

At the lowest incidence kinetic energy employed in our study $\left(E_{\mathrm{i}}=0.11 \mathrm{eV}\right)$, the trapping/desorption mechanism dominates. ${ }^{30}$ We have recorded REMPI spectra of molecules scattered in this regime for surface temperatures ranging from 140-480 K. An analysis of the spectra (see Section SIII of the ESI $\dagger$ ) reveals no significant departure from a thermal Boltzmann distribution of rotational state population and indicates nearly complete equilibration of rotational degrees of freedom to the surface temperature.

\section{B. Direct scatter channel: dependence of the rotational distribution on $\boldsymbol{E}_{\mathbf{i}}$}

The $\tilde{\mathrm{A}} \leftarrow \tilde{\mathrm{X}}\left(4_{0}^{1}\right)$ spectra of formaldehyde molecules, scattered from $\mathrm{Au}(111)$ at incidence kinetic energies over the range 0.3$1.2 \mathrm{eV}$ and probed at angles where the direct scatter channel dominates, are shown in Fig. 1. The scattered molecules are rotationally much hotter than $T_{\mathrm{s}}$ and the degree of rotational excitation increases as $E_{\mathrm{i}}$ is increased. Even from the "bird's eye" view presented in Fig. 1, a few key trends are evident in the spectra. First, as $E_{\mathrm{i}}$ is increased, spectral intensity spreads away from the band center at $\sim 28300 \mathrm{~cm}^{-1}$, and there is a general shift of intensity towards the lower frequency side of the band because the highest- $J$ progressions extend far to the red of the band center. Although the structure of the hot,

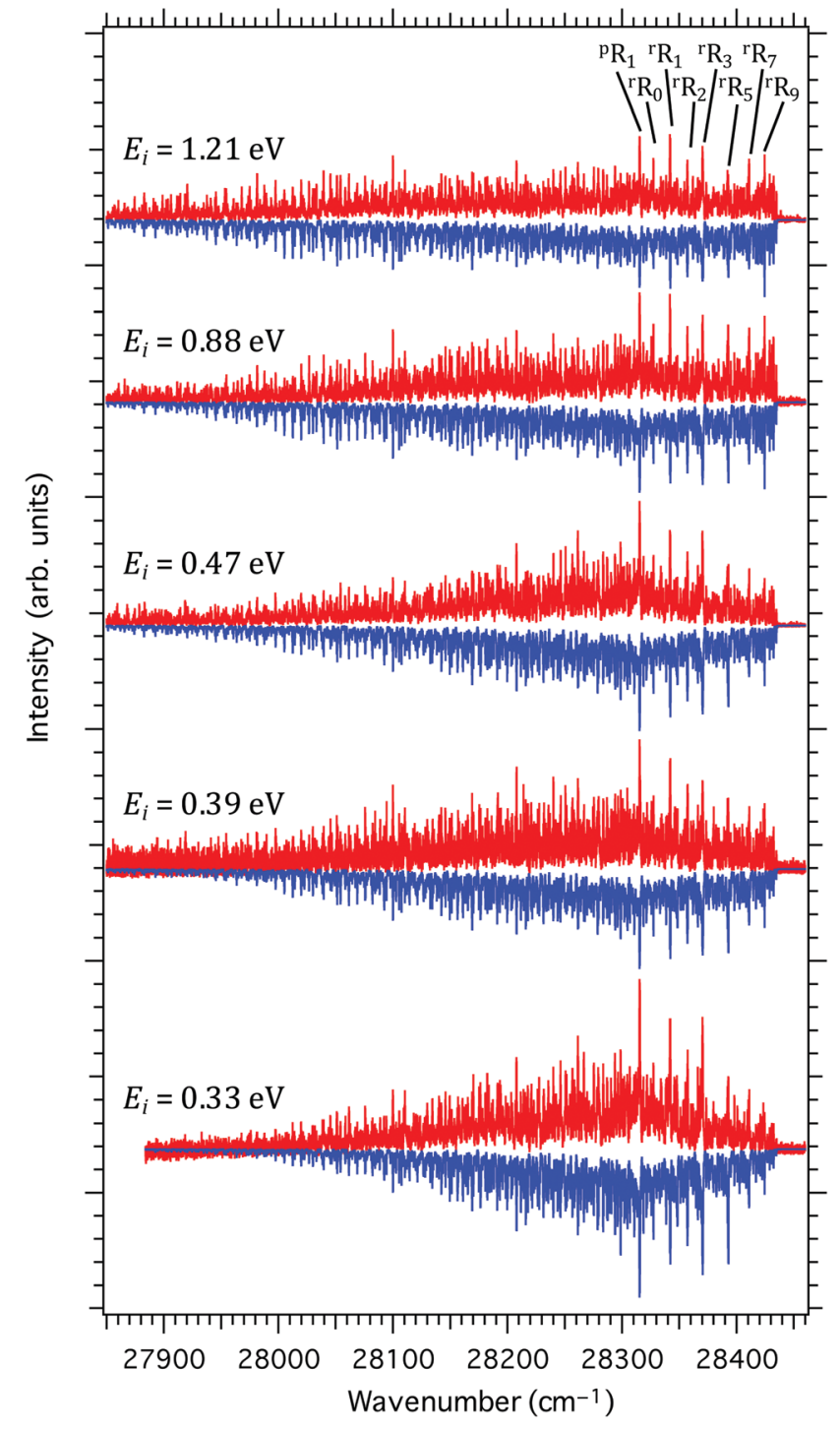

Fig. 1 The $1+1^{\prime}$ REMPI spectrum of the $\tilde{A} \leftarrow \tilde{X}\left(4_{0}^{1}\right)$ band of formaldehyde molecules, scattered from a $\mathrm{Au}(111)$ surface at $T_{\mathrm{s}}=300 \mathrm{~K}$, is shown as a function of incidence kinetic energy, $E_{\mathrm{i}}$ (red, upward directed peaks). Shown for comparison are the best-fit simulated spectra obtained using the a-axis Rainbow model (eqn (1)), which will be introduced in Section III B.2. The locations of prominent R-bandheads are labeled at the top of the figure.

asymmetric top spectra is complicated, there is a regular progression of R-bandheads that remains prominent even at the highest $E_{\mathrm{i}}$, labeled at the top of Fig. 1. (Throughout this work, we use the simplified notation ${ }^{\Delta K_{a}} \Delta J_{K_{a}^{\prime \prime}}$ to label branches of the spectrum. In cases where asymmetry splitting is resolved, we will use the more detailed notation, $\Delta K_{a}, \Delta K_{c} \Delta J_{K_{a}^{\prime \prime}, K_{c}^{\prime \prime}}\left(J^{\prime \prime}\right)$ to label individual lines.) At the lowest $E_{\mathrm{i}}$, most of the intensity is concentrated in the $K_{a}^{\prime \prime}=0-3$ bandheads. However, as $E_{\mathrm{i}}$ is increased, the relative intensity of the $K_{a}^{\prime \prime}=5-9$ bandheads gradually grows in until the progression of bandhead intensities for $K_{a}=0-9$ becomes almost flat (after taking into account the $3: 1$ ortho:para intensity alternation) at $E_{\mathrm{i}} \geq 0.88 \mathrm{eV}$. 
1. Comparison with a Boltzmann rotational distribution. Our first approach to analyzing the spectra was to compare the intensities with those predicted by a thermal Boltzmann population distribution of rotational states. However, a detailed comparison between the observed and calculated intensities reveals large, systematic discrepancies that increase with $E_{\mathrm{i}}$. The most easily recognized discrepancy is apparent in the high- $K_{a}^{\prime \prime}{ }^{\mathrm{r}} \mathrm{R}_{K_{a}^{\prime \prime}}$ bandhead region at the blue edge of the band.

Fig. 2 compares the observed $1+1^{\prime}$ REMPI spectrum in this region-obtained from molecules scattered at an incidence kinetic energy of $1.21 \mathrm{eV}$-with the spectrum simulated from the best-fit Boltzmann temperature $\left(T_{\text {rot }}=1082 \mathrm{~K}\right)$. Also shown at the bottom of Fig. 2 is a spectrum simulated using a different model for the population distribution, which we will introduce in Section III B2. The intensities obtained from the Boltzmann distribution are in very good agreement with experiment for the ${ }^{\mathrm{r}} \mathrm{R}_{5}$ bandhead at $28393 \mathrm{~cm}^{-1}$ (integrated intensity agrees to within $3 \%$ ). However, the relative intensity of the prominent ${ }^{\mathrm{r}} \mathrm{R}_{7}$ bandhead at $28411 \mathrm{~cm}^{-1}$ and the ${ }^{\mathrm{r}} \mathrm{R}_{9}$ origin at $28424 \mathrm{~cm}^{-1}$ are underestimated by the Boltzmann fit by a factor of 1.73 and 2.17 , respectively. At higher $K_{a}^{\prime \prime}$, the discrepancy becomes worse. The intense features in the observed spectrum at 28 429-28 $435 \mathrm{~cm}^{-1}$ — which arise primarily from the prominent ${ }^{\mathrm{r}} \mathrm{R}_{11}$ progression, as well as from the origins of the ${ }^{\mathrm{r}} \mathrm{R}_{12-14}$ progressions-are underestimated by the Boltzmann fit by an integrated factor of 3.4. We can be quite confident about the assignments in this region because the ${ }^{\mathrm{r}} \mathrm{R}_{K_{a}}$ " bandheads curve around to the red after $K_{a}^{\prime \prime}=13$, so that this region is relatively sparse and can only contain lines from the ${ }^{\mathrm{r}} \mathrm{R}_{10-15}$ progressions. In order to reproduce the experimentally observed relative intensities in this region, it is necessary to use a Boltzmann rotational temperature of $>3000 \mathrm{~K}$. However, the simulated band shape at this temperature exhibits wide overall disagreement with the rest of the observed spectrum. The enhanced intensity in the high- $K_{a}{ }^{\prime \prime} \mathrm{R}_{K_{a}}$ ' bandhead region (relative to that predicted for a Boltzmann distribution) is reproducible across a wide range of incidence kinetic energies and increases monotonically with $E_{\mathrm{i}}$.

The low-frequency tail of the band below $28200 \mathrm{~cm}^{-1}$, which contains intensity primarily from ${ }^{\mathrm{p}} \mathrm{P}_{K_{a}^{\prime \prime}}$ branches, is another relatively sparse region that permits a detailed line-by-line analysis of the relative intensities. In Fig. 3, the observed spectrum in this region obtained from a scattered beam $\left(E_{\mathrm{i}}=1.21 \mathrm{eV}\right)$ is compared with the simulated spectrum from the best-fit Boltzmann distribution. Again, a simulation from a second model, which we will introduce in Section III B2, is also included in the figure. The observed spectrum contains prominent progressions that can be assigned to the ${ }^{{ }^{P}} P_{9}$ and ${ }^{{ }^{P}} P_{11}$ branches, labeled in the figure. However, the Boltzmann fit moderately underestimates the contribution of ${ }^{\mathrm{P}} \mathrm{P}_{9}$ lines-particularly at low $J^{\prime \prime}$-and grossly underestimates the contribution of ${ }^{\mathrm{P}} \mathrm{P}_{11}$. For example, the spectrum displays significantly more intensity than the Boltzmann fit at $28110.6 \mathrm{~cm}^{-1}$ where the ${ }^{\mathrm{P}} \mathrm{P}_{9}(9)$ line is predicted. The features observed at $28076.0 \mathrm{~cm}^{-1}$ and $28100.1 \mathrm{~cm}^{-1}$ are significantly blue-shifted (by 0.25 and $0.1 \mathrm{~cm}^{-1}$, respectively) in the Boltzmann simulation, which is consistent with missing intensity from the ${ }^{\mathrm{P}} \mathrm{P}_{9}$ (15) and ${ }^{\mathrm{P}} \mathrm{P}_{9}(11)$ lines. Finally, the relative intensity of the features assignable to ${ }^{\mathrm{P}} \mathrm{P}_{11}(11-16)$ is strikingly absent from the Boltzmann fit.

There are other features that appear with high relative intensity in the Boltzmann fit, but are not observed in the spectrum. Among the most prominent of these features are the lines arising from ${ }^{\mathrm{P}} \mathrm{P}_{1}$ (32-38), which are labeled in Fig. 3 . The intensity of the features that contain contributions from ${ }^{\mathrm{P}} \mathrm{P}_{1}(33-35)$ at $28082.1,28092.6$, and $28102.8 \mathrm{~cm}^{-1}$ are notably stronger in the Boltzmann fit than in the observed spectrum. Also, the Boltzmann fit predicts intensity at $28049.7 \mathrm{~cm}^{-1}$ and $28101.3 \mathrm{~cm}^{-1}$ that arises from the ${ }^{\mathrm{P}} \mathrm{P}_{1}(38)$ and ${ }^{\mathrm{P}, \mathrm{P}} \mathrm{P}_{3,30}$ (33) lines, respectively, whereas there is almost no intensity in the observed spectrum at these frequencies.

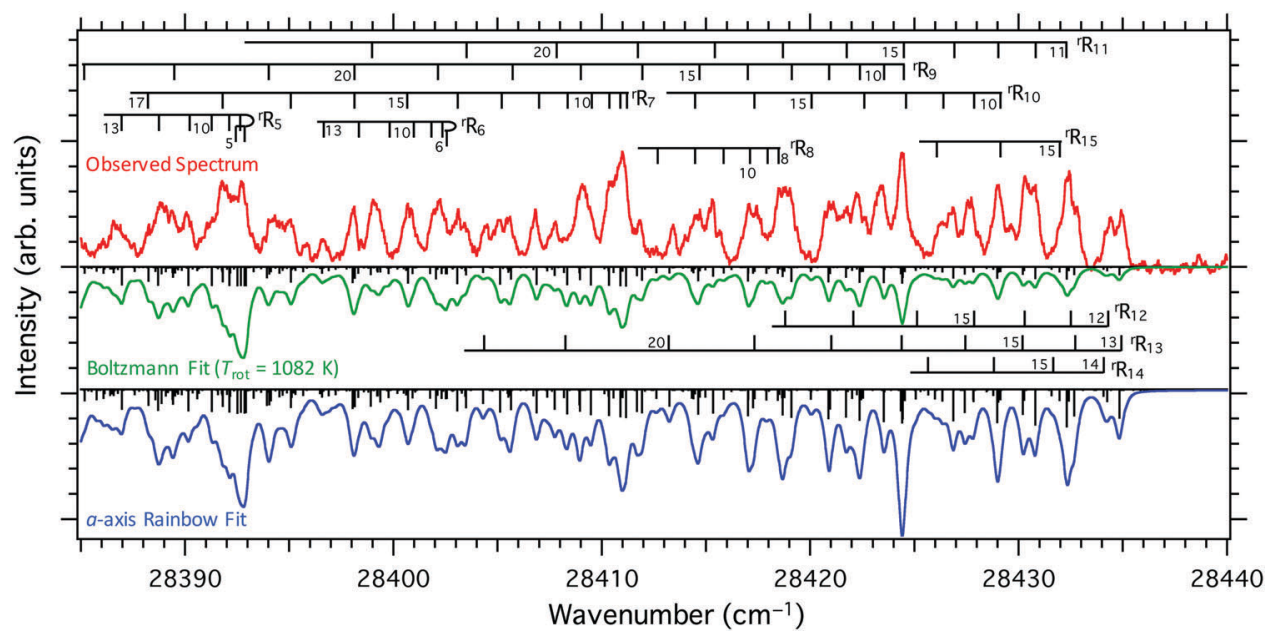

Fig. 2 The high- $K_{a}{ }^{\prime \prime}{ }^{\mathrm{r}} \mathrm{R}_{K_{a}}$ " bandhead region of the REMPI spectrum of the $\tilde{\mathrm{A}} \leftarrow \tilde{\mathrm{X}}\left(4_{0}^{1}\right)$ band of formaldehyde molecules, directly scattered from a Au(111) surface at $T_{\mathrm{s}}=300 \mathrm{~K}$ and $E_{\mathrm{i}}=1.21 \mathrm{eV}$ is shown (top spectrum, red, upward directed peaks). Shown for comparison are the best-fit simulated spectra obtained from a Boltzmann model (middle spectrum, green, downward directed peaks) and from the $a$-axis rainbow model (eqn (1)), which will be introduced in Section III B2. The ${ }^{\mathrm{r}} \mathrm{R}_{5-15}$ branch transitions are labeled. 


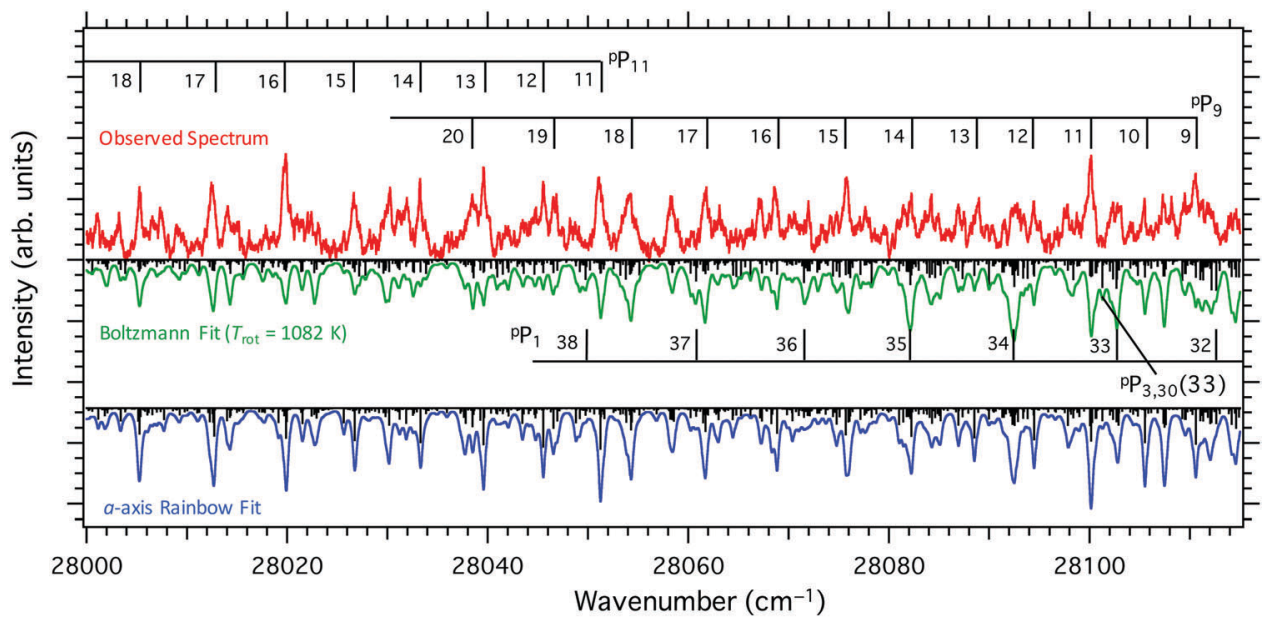

Fig. 3 A portion of the ${ }^{\mathrm{P} P}$ region of the REMPI spectrum of the $\tilde{A} \leftarrow \tilde{X}\left(4_{0}^{1}\right)$ band of formaldehyde molecules, directly scattered from a Au(111) surface at $T_{\mathrm{s}}=300 \mathrm{~K}$ and $E_{\mathrm{i}}=1.21 \mathrm{eV}$ is shown (top spectrum, red, upward directed peaks). Shown for comparison are the best-fit simulated spectra obtained from a Boltzmann model (middle spectrum, green, downward directed peaks) and from the $a$-axis rainbow model (eqn (1)), which will be introduced in Section III B2. Selected branches and transitions are labeled.

2. $\boldsymbol{a}$-axis rotational rainbow model. The lower states involved in the series of transitions labeled in Fig. 3 are similar in total rotational energy. (The $J_{K_{a}^{\prime \prime}}^{\prime \prime}=11_{11}-18_{11}$ series has $1141-1395 \mathrm{~cm}^{-1}$ of rotational energy, whereas the $J_{K_{a}^{\prime \prime}}^{\prime \prime}=32_{1}-38_{1}$ series has $1231-1719 \mathrm{~cm}^{-1}$ of rotational energy.) However, the distribution of rotational energy about the distinct internal axes of rotation is very different in these two series. In a near prolate top molecule, $K_{a}$ is an approximately good quantum number that accurately describes the projection of angular momentum about the $a$-axis (the CO bond axis). The low- $J$ members of the $K_{a}=11$ manifold have almost all of their angular momentum about the $a$-axis ("twirling" motion), whereas the high- $J$ levels of the $K_{a}=1$ manifold have almost all of their angular momentum distributed about the $b$ - and $c$-axes ("tumbling" or "cartwheeling" motion).

The analysis presented in Section III B1 suggests that the rotational population distribution in the scattered molecular beam deviates from a thermal distribution in a way that favors "twirling" motion with $K_{a}$ in the range 7-13, over "tumbling/ cartwheeling" motion with the same amount of energy. Deviations from a Boltzmann rotational distribution appear quite commonly in experiments on direct scattering of diatomic molecules from surfaces. ${ }^{2,9,17}$ Such effects are orientation dependent ${ }^{12-16,35}$ and can often be interpreted in terms of a "rotational rainbow" effect, in which dependence of the potential energy surface on molecular orientation gives rise to one or more singularities in the classical distribution of angular momentum in the scattering product.

In diatomic molecule experiments, the observed rotational rainbow population distribution often resembles a Boltzmann distribution with an added Gaussian term, centered at the rainbow energy. ${ }^{18,36}$ In formaldehyde, there are three distinct projections of the angular momentum onto the molecular body frame and the problem is more complicated. Based on the results of our analysis in Section III B1, we attempt a model that partitions the rotational energy into two parts: $E_{a}$ ( $a$-axis rotational energy) and $E_{b c}$ (combined $b$ - and $c$-axis rotational energy). We add a Gaussian contribution to the $a$-axis distribution function to obtain

$$
P_{i}=N g_{i} \mathrm{e}^{-E_{b c, i} / k T_{b c}}\left(\mathrm{e}^{-E_{a, i} / k T_{a}}+S \mathrm{e}^{-\left(E_{0}-E_{a, i}\right)^{2} / \sigma_{a}^{2}}\right) .
$$

In eqn (1), $P_{i}$ is the population of state $i, N$ is a normalization factor, and $g_{i}$ is a degeneracy factor that includes both $M_{J}$ and nuclear spin state degeneracy. The first factor in eqn (1) describes the distribution of the $E_{b c}$ partition of the energy, and the second factor describes the distribution of the $E_{a}$ partition. $T_{a}$ and $T_{b c}$ are effective "temperatures" for the two types of energy and $S$ parameterizes the extent to which population is distributed non-thermally in $E_{a} . E_{0}$ corresponds to the $a$-axis rotational energy of the rainbow, and $\sigma_{a}$ is a width parameter that describes the sharpness of the rainbow. Note that eqn (1) converges to a Boltzmann distribution in the limit $T_{a}=T_{b c}$ and $S=0$. Neglecting the relatively small contributions of asymmetry splitting and centrifugal distortion, we set

$$
\begin{gathered}
E_{a}=A K_{a}{ }^{2} \\
E_{b c}=E_{\mathrm{rot}}-A K_{a}{ }^{2},
\end{gathered}
$$

where the rotational constant $A \approx 9.406 \mathrm{~cm}^{-1}$. In order to reduce correlation between the fit parameters, we constrain $T_{a}=T_{b c}$ (i.e. we used the same "Boltzmann temperature" for each degree of freedom), and we constrain the Gaussian width parameter to $\sigma_{a}=850 \mathrm{~cm}^{-1}$, which is the value that gives the best fit at $E_{\mathrm{i}}=1.21 \mathrm{eV}$, where the observed deviation from thermal distribution is greatest.

The spectrum was recorded several times at each $E_{\mathrm{i}}$ shown in Fig. 1, and each spectrum was fit individually to eqn (1). The average and standard deviation of the fit parameters obtained at each $E_{\mathrm{i}}$ are shown in Fig. 4(A-C) and are tabulated in Table SIII of the ESI. $\dagger$ Also shown in Fig. 4(D) is the percent improvement in 

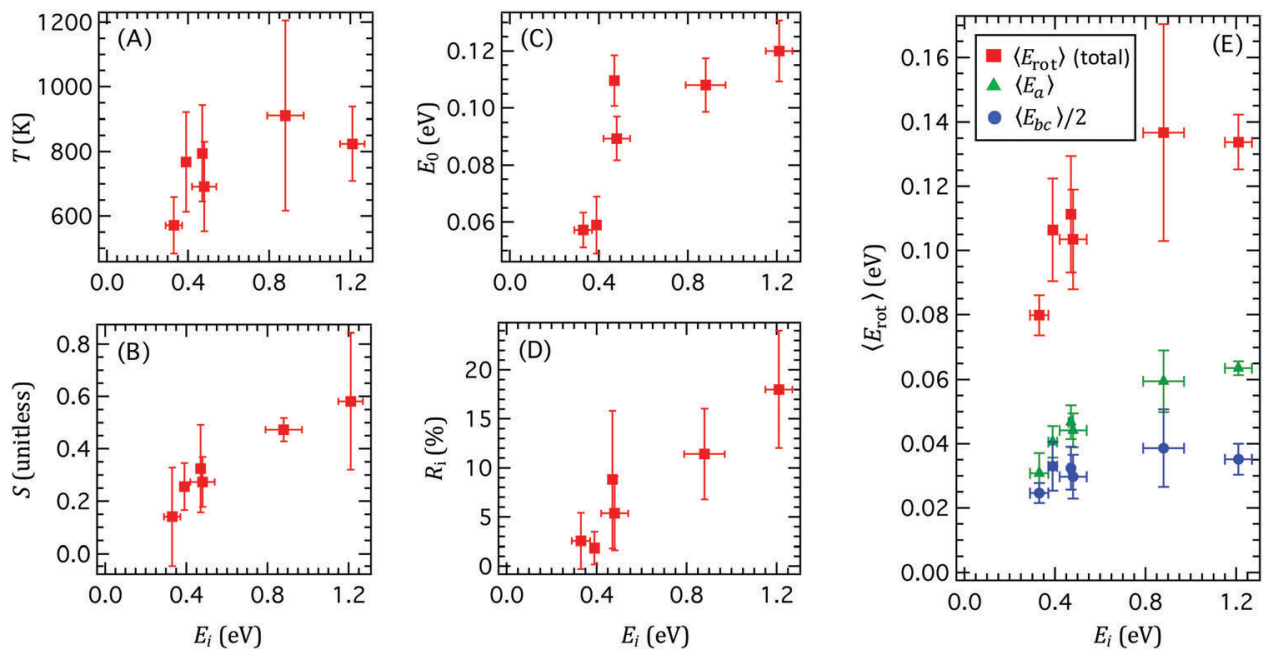

Fig. 4 The best-fit parameters obtained by fitting the spectra of scattered molecules to eqn (1), with the constraints $T_{b c}=T_{a}=T$ and $\sigma_{a}=850 \mathrm{~cm}^{-1}$, are shown as a function of incidence kinetic energy, $E_{\mathrm{i}}$, in panels (A), (B) and (C). Panel (D) shows the average percent improvement, $R_{\mathrm{i}}$, in the root mean square error of the fit, $R_{\mathrm{fit}}$, relative to the RMSE obtained from a fit to a Boltzmann temperature, $R_{\mathrm{b}} . R_{\mathrm{i}}=\left(1-R_{\mathrm{b}} / R_{\mathrm{fit}}\right) \times 100 \%$. Panel $(\mathrm{E})$ shows the expectation value, obtained from the fit model, for total rotational excitation, and for the components $E_{a}$ and $E_{b c}$.

the root mean square error (RMSE) that is obtained from the fit to eqn (1), relative to the RMSE obtained from a fit to a Boltzmann distribution. Shown in Fig. 4(E) is the expectation value of rotational energy, $\left\langle E_{\text {rot }}\right\rangle$, obtained from the model at each incidence kinetic energy as well as the individual contributions, $\left\langle E_{a}\right\rangle$, and $\left\langle E_{b c}\right\rangle$. The best-fit spectrum obtained at each incidence energy is compared with the observed spectrum in Fig. 1, and the result obtained at $E_{\mathrm{i}}=1.21 \mathrm{eV}$ is included in Fig. 2 and 3.

Several trends are evident in the fit results. As $E_{\mathrm{i}}$ is increased, there is a slight trend towards higher $T$ parameter (Fig. 4(A)), consistent with a general increase in both $\left\langle E_{a}\right\rangle$ and $\left\langle E_{b c}\right\rangle$. The average rotational energy obtained from the model for the scattered beam increases approximately linearly over the range $E_{\mathrm{i}}=0.33-0.8 \mathrm{eV}$ with a slope of $\left\langle E_{\text {rot }}\right\rangle \approx 0.24 E_{\mathrm{i}}$, and appears to exhibit some degree of saturation above $E_{\mathrm{i}}=0.8 \mathrm{eV}$ (Fig. 4(E)). In addition, the $a$-axis rainbow model reveals an increasing propensity for energy to be channeled into $a$-axis rotation as $E_{\mathrm{i}}$ is increased. This trend is evident from the increasing ratio between $\left\langle E_{a}\right\rangle$ and $\left\langle E_{b c}\right\rangle$ (Fig. 4(E)) and leads to a linearly increasing trend in the best-fit amplitude, $S$, of the non-thermal Gaussian term, which has a near-zero intercept at $E_{\mathrm{i}}=0$ (Fig. 4(B)). The fitted values for $E_{0}$ (Fig. $4(\mathrm{C})$ ) indicate that the maximum in the nonthermal Gaussian contribution to the population distribution shifts from $K_{a} \approx 7$ at $E_{\mathrm{i}}=0.4 \mathrm{eV}$ to $K_{a} \approx 10$ at $E_{\mathrm{i}}=1.2 \mathrm{eV}$.

A consequence of the increasing importance of the nonthermal contribution to the population distribution is that the failure of the Boltzmann model to reproduce the observed spectrum increases strikingly as $E_{\mathrm{i}}$ is increased. At low incidence kinetic energy $\left(E_{\mathrm{i}}<0.4 \mathrm{eV}\right)$, the observed spectrum is well reproduced by the Boltzmann fit, and the improvement of the fit error that is obtained by including the non-thermal Gaussian component of eqn (1) is negligible (Fig. 4(D)). However, at higher $E_{\mathrm{i}}$, the Boltzmann fit fails to reproduce the spectral intensities (as shown in Fig. 2 and 3) and the improvement in the overall
RMSE obtained by including the Gaussian term reaches almost $20 \%$ at $E_{\mathrm{i}}=1.2 \mathrm{eV}$.

From a comparison of the observed spectra in Fig. 2 and 3 to the Boltzmann fit and the $a$-axis rainbow model fit, it is clear that the $a$-axis rainbow model provides significantly better agreement with experiment. The integrated intensities in the ${ }^{r} R_{5},{ }^{r} R_{7}$, and ${ }^{r} R_{9}$ bandheads obtained from the rainbow model all agree with experiment to within $16 \%$, and the integrated intensity over the origins of the ${ }^{\mathrm{r}} \mathrm{R}_{11-14}$ progressions (28 429-28 $435 \mathrm{~cm}^{-1}$ ) agrees with experiment to within $4 \%$ (Fig. 2). The $a$-axis rainbow fit also correctly reproduces the observed relative intensities in the ${ }^{\mathrm{P}} \mathrm{P}_{11}$ (11-16) lines between 28000 and 28060 , and includes features from the ${ }^{\mathrm{P}} \mathrm{P}_{9}$ branch such as the intensity due to ${ }^{\mathrm{P}} \mathrm{P}_{9}(9)$ at $28110.6 \mathrm{~cm}^{-1}$ (Fig. 3). Unlike the Boltzmann fit, the $a$-axis rainbow fit does not place too much intensity in the ${ }^{\mathrm{P}} \mathrm{P}_{1}(33-38)$ lines or in the ${ }^{\mathrm{p}, \mathrm{P}} \mathrm{P}_{3,30}$ (33) line (discussed in Section III B1). Furthermore, the $a$-axis rainbow fit correctly reproduces (to within $0.05 \mathrm{~cm}^{-1}$ ) the center frequencies of the observed features at $28076.0 \mathrm{~cm}^{-1}$ and $28100.1 \mathrm{~cm}^{-1}$, which are shifted relative to the Boltzmann fit due to contributions from ${ }^{{ }^{\mathrm{P}}} \mathrm{P}_{9}(15)$ and ${ }^{\mathrm{P}} \mathrm{P}_{9}(11)$.

In Fig. 5 , the sum of the population in each $K_{a}$ manifold (divided by the $g_{\text {ns }}$ nuclear spin degeneracy) obtained from the fit to the $a$-axis rainbow model is plotted versus $E_{a}=A K_{a}^{2}$ as a function of $E_{\mathrm{i}}$. At the lowest incidence energy, the population decreases approximately exponentially with $E_{a}$, as expected in a thermal distribution. As $E_{\mathrm{i}}$ is increased, a strong shoulder grows in at $\sim 0.12 \mathrm{eV}$, due to the increasing importance of the Gaussian term in eqn (1). The population in $K_{a}=0$ is a factor of two below the trend because this manifold lacks the two-fold $k= \pm\left|K_{a}\right|$ degeneracy (i.e. there are no asymmetry doublets).

\section{Classical scattering simulation}

To aid in the interpretation of our results, we have calculated classical scattering trajectories. We treat the formaldehyde molecule as a rigid rotor with the geometry given by Burleigh et al. ${ }^{37}$ 
$K_{a}$

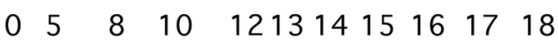

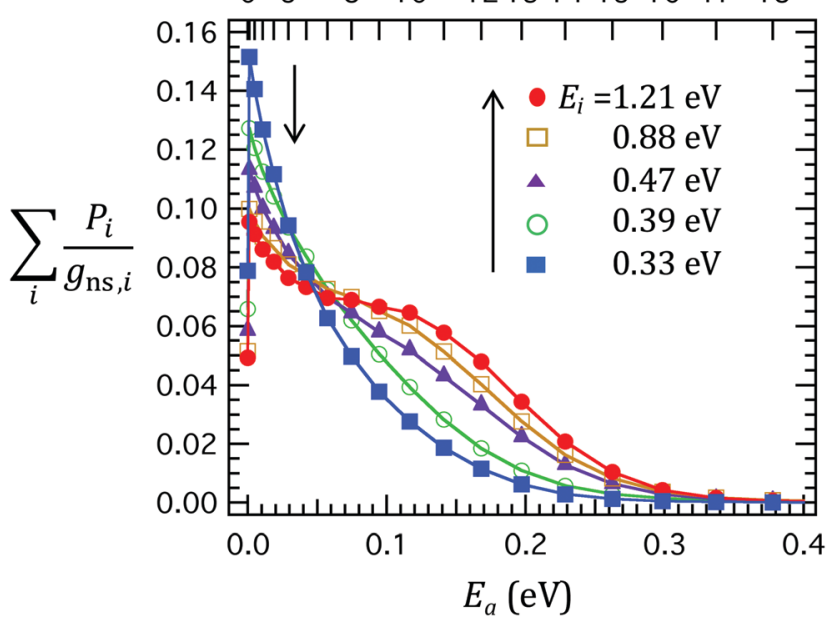

Fig. 5 The population within each $K_{a}$ manifold, obtained from the a-axis rainbow model fit to the direct-scatter REMPI data is divided by the nuclear spin degeneracy $\left(g_{\mathrm{ns}}\right)$ and plotted as a function of $E_{\mathrm{i}}$. The total population at each $E_{\mathrm{i}}$ is normalized to one. The population in $K_{a}=0$ is a factor of two below the trend because this manifold lacks the two-fold $k= \pm\left|K_{a}\right|$ degeneracy.

We approximate the surface as a flat harmonic oscillator with mass $197 \mathrm{amu}$ and the Debye frequency of gold $\left(\omega=k \Theta_{\mathrm{D}} / h\right.$ with $\left.\Theta_{\mathrm{D}}=170 \mathrm{~K}\right)$. We tried two different simple models for the molecule-surface interaction. First, we used a purely repulsive hard-sphere potential ("hs") with a hard wall at the van der Waals radius of each atom. Next, we used an empirical Lennard-Jones 6-12 potential ("L-J"), with parameters taken from the UFF force field. $^{38}$ The UFF van der Waals interaction underestimates the measured $^{30}$ and calculated ${ }^{39-41}$ binding energy of formaldehyde on $\mathrm{Au}(111)$ by more than an order of magnitude, so we scale the potential by a factor of 41 in order to bring the optimized binding energy into agreement with the measured value $(0.31 \mathrm{eV}) .^{30}$

The trajectories were calculated at normal incidence angle and random incidence orientation, over a range of incidence kinetic energies $\left(E_{\mathrm{i}}=0.33-1.2 \mathrm{eV}\right)$. The surface was initially at rest and the molecule was initially at a distance of $z_{0}=10 \AA$ from the surface with zero incident angular momentum. We integrated $10 \mathrm{k}$ trajectories for $2.4 \mathrm{ps}$ at each $E_{\mathrm{i}}$. For the L-J potential, any trajectory in which the molecule remained within $8 \AA$ of the surface after 2.4 ps was labeled a "trapping" trajectory and was discarded. At $E_{\mathrm{i}}=0.33 \mathrm{eV}, 28 \%$ of trajectories were discarded on this basis. The proportion of "trapping" trajectories drops to $15 \%$ at $E_{\mathrm{i}}=0.47 \mathrm{eV}$ and to $2.1 \%$ at $E_{\mathrm{i}}=1.2 \mathrm{eV}$. This trend moderately underestimates the observed trapping probability, ${ }^{30}$ which drops from $57 \%$ at $E_{\mathrm{i}}=0.32 \mathrm{eV}$ to $25 \%$ at $E_{\mathrm{i}}=0.58 \mathrm{eV}$.

After exiting the surface potential, the rotational motion of the directly scattered asymmetric top molecules propagates according to Newton's equations of torque-free motion:

$$
\begin{gathered}
I_{a a} \dot{\omega}_{a}=\omega_{b} \omega_{c}\left(I_{b b}-I_{c c}\right) \\
I_{b b} \dot{\omega}_{b}=\omega_{c} \omega_{a}\left(I_{c c}-I_{a a}\right) \\
I_{c c} \dot{\omega}_{c}=\omega_{a} \omega_{b}\left(I_{a a}-I_{b b}\right) .
\end{gathered}
$$

In the general case, eqn (2) leads to precession of the angular velocity about the three body-fixed internal axes, which "scrambles" the memory of the body-fixed components of the torque induced by the surface scattering event. However, for a near-prolate symmetric top, $I_{b b}-I_{c c} \approx 0$, and eqn (2a) reduces to $I_{a a} \dot{\omega}_{a} \approx 0$. As a consequence, $\omega_{a}$ (and $E_{a}=\frac{1}{2} \omega_{a}^{2} I_{a a}$ ) are approximately conserved constants of motion, as is the quantity $E_{\mathrm{rot}}-E_{a}=E_{b}+E_{c}$. However, the individual components $E_{b}$ and $E_{c}$ oscillate in time due to precession about the near-symmetry axis.

Fig. 6 shows the final average rotational energy about each internal axis of rotation, calculated from the two potentials as a function of $E_{\mathrm{i}}$. The calculated values may be compared directly with the values from the experimental fits, shown in Fig. 4(E). Although the calculations overestimate the overall amount of rotational excitation, relative to the experimental fit, by a factor of $\sim 2$, they nevertheless reproduce key qualitative features of the experimental observation. Most notably, regardless of whether an attractive potential is used, the calculation predicts that $\left\langle E_{a}\right\rangle$ excitation is greater than $\left\langle E_{b}\right\rangle$ or $\left\langle E_{c}\right\rangle$ excitation by an average factor of $2\left\langle E_{a}\right\rangle /\left(\left\langle E_{b}\right\rangle+\left\langle E_{c}\right\rangle\right)=1.6$ (L-J) or 1.3 (hs), compared to an experimental value of 1.5 . When the purely repulsive hard sphere potential is used, rotational excitation increases linearly with $E_{\mathrm{i}}$, but when the Lennard-Jones potential is used, the calculation also qualitatively reproduces the experimental observation that $\left\langle E_{a}\right\rangle$ exhibits some degree of saturation at higher $E_{\mathrm{i}}$. These results suggest that the propensity to excite $a$-axis rotation is a simple consequence of the three-dimensional repulsive van der Waals shape of the formaldehyde molecule, whereas the saturation effect may be a signature of dynamical steering due to the orientation dependence of the attractive potential.

The top half of Fig. 7 shows the distribution of rotational energy about the internal axes of rotation calculated using the L-J potential with $E_{\mathrm{i}}=1.2 \mathrm{eV}$. The $E_{b}$ and $E_{c}$ distributions can be approximated by an exponential decay. On the other hand, the $E_{a}$ distribution exhibits a distinct non-exponential component, which gives rise to a broad feature between $0.15-0.4 \mathrm{eV}$. The calculated rotational distributions are in qualitative agreement

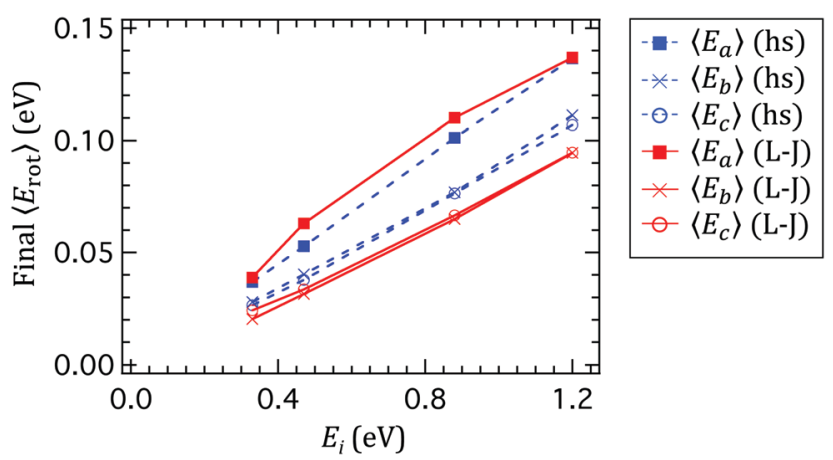

Fig. 6 The expectation value for rotational excitation about each principal internal axis of the formaldehyde molecule, obtained from classical scattering trajectory calculations, as a function of incidence kinetic energy, $E_{i}$, at normal scattering angle. Two interaction potentials were used: a repulsive hardsphere potential (hs) and an empirically scaled Lennard-Jones potential (L-J). See text for further details. 

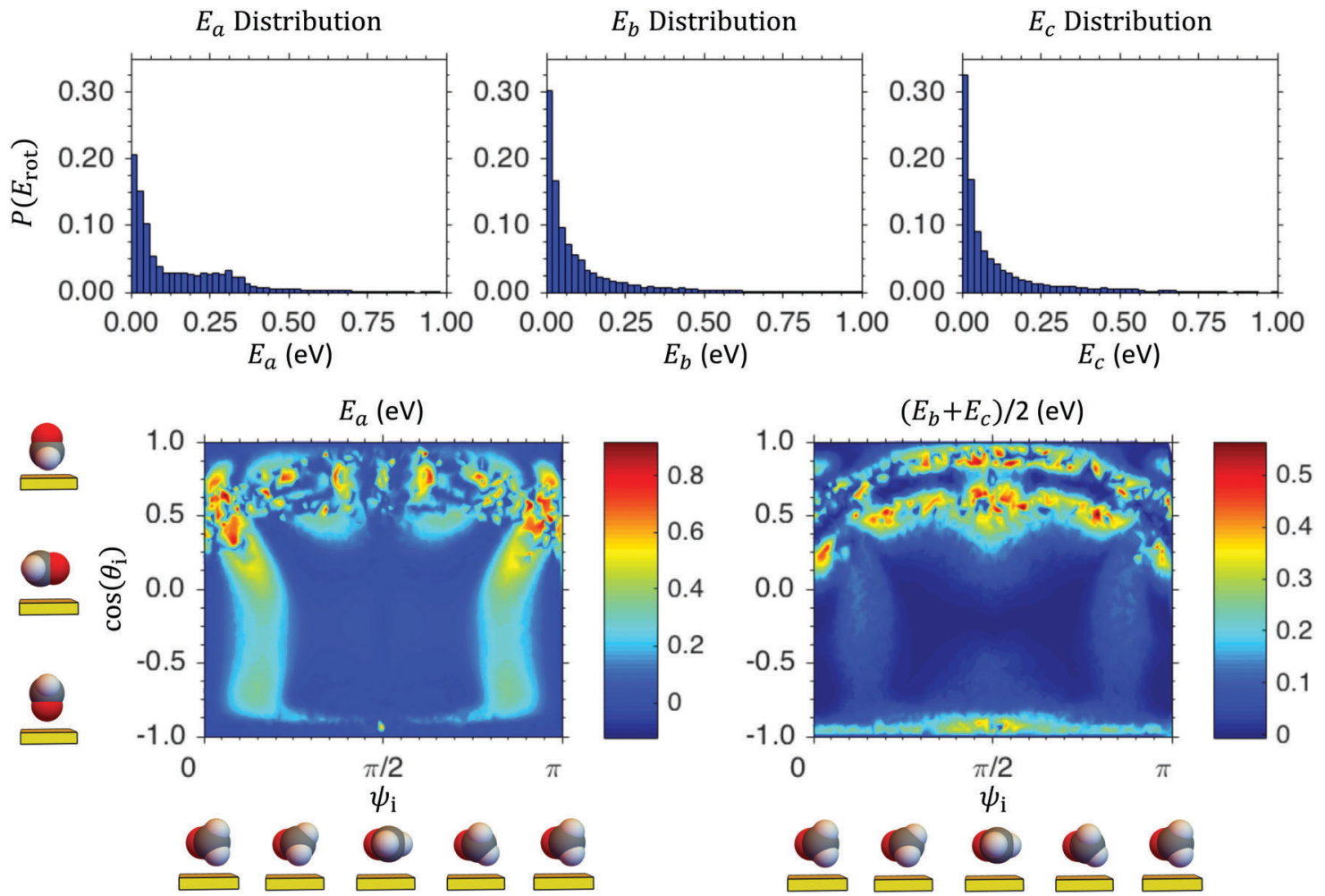

Fig. 7 Upper panels display the normalized distribution of rotational energy about each principal internal axis of the formaldehyde molecule, obtained from a classical scattering trajectory calculation using the L-J potential at $E_{\mathrm{i}}=1.2 \mathrm{eV}$, at normal scattering angle. The bottom panels show the rotational energy components, $E_{a}$ and $\left(E_{b}+E_{c}\right) / 2$, as a function of incidence orientation, described by the Euler angles $\theta_{\mathrm{i}}$ and $\psi_{\mathrm{i}}$. See text for further details

with the distributions obtained from our fit model. Namely, the $E_{b}$ and $E_{c}$ distributions resemble an exponential Boltzmann population distribution whereas the $E_{a}$ distribution resembles an exponential decay plus an added Gaussian component (see Fig. 5).

The classical trajectory calculation is not in complete quantitative agreement with the experimental fit model. The calculation overestimates the average overall rotational excitation, and the calculated non-Boltzmann hump in the $E_{a}$ distribution is broader and extends to higher energy than that of the fit model. The trajectory calculation places the center of the hump at $0.30 \mathrm{eV}$ whereas the fit model places the center at $0.12 \mathrm{eV}$. However, since the trajectory calculation uses only a crude empirical potential energy surface, it is unlikely to capture the orientation dependence of the potential accurately and complete quantitative agreement cannot be expected. Theoretical investigations involving a more sophisticated $a b$ initio potential energy surface are merited, but are beyond the scope of the current work. Nevertheless, since the classical trajectories reproduce key qualitative features of the experiment, they provide clues as to the likely origin of these features.

The bottom half of Fig. 7 shows the components, $E_{a}$ and $\left(E_{b}+E_{c}\right) / 2$, of rotational energy, calculated as a function of the incident Euler angles, $\theta_{\mathrm{i}}$ and $\psi_{\mathrm{i}}$. In our chosen coordinate system, $\theta_{\mathrm{i}}$ represents the polar angle between the molecular CO-bond symmetry axis and the surface normal, and $\psi_{i}$ represents the angle between the surface normal and its orthogonal projection onto the $\mathrm{CH}_{2}$ plane. See cartoons in Fig. 7 for clarification. Normal angle scattering of a $C_{2 \mathrm{v}}$ molecule from a flat, structureless surface is totally symmetric in the molecule-fixed axis frame with respect to the third Euler angle, $\phi$, which is omitted from the figure. The $b$ - and $c$-axis projections of the rotational energy are maximally excited at incidence orientations where the $\mathrm{CO}$ axis is nearly (but not exactly) along the surface normal $\left(\theta_{\mathrm{i}} \approx 0\right.$ or $\left.\pi\right)$, and are minimally excited when the incident $\mathrm{CO}$ axis orientation is "flat-on" $\left(\theta_{\mathrm{i}} \approx \pi / 2\right)$. At incidence angles near $\theta_{\mathrm{i}} \approx 0\left(\mathrm{CH}_{2}\right.$ group oriented toward the surface), the prevalence of "multi-bounce" collisions, where the two hydrogen atoms strike the surface in rapid succession, give rise to complicated structure in all three components of $E_{\mathrm{rot}}$. Apart from a relatively narrow region near $\theta_{\mathrm{i}}=\pi$, the plot of $\left(E_{b}+E_{c}\right) / 2$ versus incident orientation does not reveal any widespread regions over which the function exhibits a smooth maximum.

In contrast, $E_{a}$ exhibits broad regions of incidence orientation space over which it is excited to approximately the same value. Most strikingly, when $\pi / 20<\psi_{\mathrm{i}}<\pi / 4$ or $3 \pi / 4<\psi_{\mathrm{i}}<19 \pi / 20$, excitation to $E_{a} \approx 0.3 \mathrm{eV}$ is highly likely, regardless of the value of $\theta_{\mathrm{i}}$. The explanation is simple. A large degree of $a$-axis excitation results whenever one of the hydrogens strikes the surface at a skew angle of $\psi_{\mathrm{i}}$, regardless of the polar angle $\theta_{\mathrm{i}}$ between the CO axis and the surface normal. This occurs even at incident orientations in which the $\mathrm{CH}_{2}$ group is initially pointed away from the surface $\left(\theta_{\mathrm{i}}<0\right)$ due to "double-bounce" collisions in which the $\mathrm{O}$ and $\mathrm{CH}_{2}$ moieties strike the surface in rapid succession. 
Since this behavior arises over a wide swath of orientation space, excitation probability accumulates at $E_{a} \approx 0.3 \mathrm{eV}$ over many trajectories and gives rise to the distinctly non-Boltzmann propensity for high $K_{a}$ excitation which is observed. This explanation allows us to interpret the effect in terms of a sterically induced rotational rainbow scattering phenomenon. Because there is a wide region of orientation space over which the Jacobian $\partial E_{a} / \partial\left(\psi_{\mathrm{i}}, \theta_{\mathrm{i}}\right)$ is approximately zero (and a long curve over which $\partial E_{a} / \partial \psi_{\mathrm{i}}$ is rigorously zero), the classical probability distribution for $E_{a}$ as a function of $\psi_{\mathrm{i}}$ contains singularities for excitation to the local extrema in $E_{a}$.

\section{Discussion}

\section{A. Possible sources of error in the analysis}

Alternative explanations for the apparent non-Boltzmann intensities must be considered and excluded. In ref. 32 we characterized our REMPI scheme and found no significant discrepancies between our measured relative intensities and the relative intensities in the calculated absorption spectrum, to within an uncertainty of $\sim 20 \%$. Although the spectra analyzed in that work were obtained at rotational temperatures of $300 \mathrm{~K}$ or less, and did not include significant intensity from transitions involving states with $K_{a}^{\prime \prime} \geq 9$, our results allow us to rule out the possibility that the observed intensity at higher $K_{a}^{\prime \prime}$ arises from artifacts such as $K_{a}$-dependent ionization cross sections or $K_{a}$-dependent dissociative lifetimes of the intermediate $\tilde{A}$ state levels. The intensities from $K_{a}^{\prime \prime}=9$ transitions measured in the trapping/desorption channel (top spectrum, Fig. SI, ESI $\dagger$ ) agree with the Boltzmann simulation to within $10 \%$. Furthermore, the magnitude of the observed non-thermal relative intensities in the direct scatter channel grows in as a function of $E_{\mathrm{i}}$, and is therefore consistent with a population effect and not with incorrect relative line intensities. In addition, the high- $K_{a}^{\prime \prime}$ populations that one obtains through analysis of the ${ }^{\mathrm{r}} \mathrm{R}_{K_{a}^{\prime \prime}}$ branches matches that which one obtains from the ${ }^{\mathrm{p}} \mathrm{P}_{K_{a}^{\prime \prime}}$ branches, although these transitions involve Ã-state intermediate levels with different $K_{a}{ }^{\prime}$. The results that we report by fitting the entire band are reproduced when either the ${ }^{r} \mathrm{R}$ or ${ }^{\mathrm{P}} \mathrm{P}$ region is fitted independently.

Another possible source of intensity error in our measurement arises from the fact that we have not performed exhaustive rotationally-resolved measurement of the speed distribution of scattered molecules. Therefore, our population estimates are based on scattered beam density measurements that have not been converted to flux, and are only valid in the limit $\Delta v /\langle v\rangle \ll 1$ (i.e. when the velocity spread is small compared with the mean velocity.) Experimental limitations make it difficult to perform precise rotationally resolved time-of-flight measurements. However, we have made coarse speed measurements for a representative sample of rotational states differing in $E_{\text {rot }}$ and $K_{a}$. The results, which are detailed in Section SV of the ESI, $\dagger$ provide an upper limit for $\Delta v /\langle v\rangle<10 \%$ for the rotational state dependence of the average scattered velocity at $E_{\mathrm{i}}=1.21 \mathrm{eV}$, which is far too small to account for deviation of the observed intensities from a Boltzmann model.
We have also investigated the effect of laser polarization, and we find no difference in relative intensities when the dye laser polarization is rotated from horizontal to vertical polarization. Orientation of the angular momentum due to the surface is likely scrambled due to nuclear hyperfine dephasing before the scattered molecules reach the detection region.

\section{B. Trapping probability at high $E_{\mathrm{i}}$}

Shown in the top panel of Fig. 8 is the measured trapping probability of formaldehyde on the $\mathrm{Au}(111)$ surface as a function of incidence energy at normal incidence angle, reported in ref. 30. Following Fig. 6 of ref. 30, the trapping probabilities from three other weakly bound, non-activated scattering systems (with binding energies in the range $0.18-0.33 \mathrm{eV}$ ) of similar molecular mass (28-30 amu) and surface atom mass (195-197 amu) are shown for comparison. In a hard cube model, ${ }^{42-44}$ these systems are expected to exhibit similar trapping probability. However, in comparison with the diatomic molecules NO and CO, formaldehyde exhibits a significantly higher trapping probability. The shift of $\sim 0.3 \mathrm{eV}$ in the initially decaying portion of the curve is much too large to be explained by differences in binding energy. Strikingly, formaldehyde exhibits $25 \%$ trapping probability at an incidence energy of $0.58 \mathrm{eV}$-more than an order of magnitude higher than the trapping probability of NO at similar incidence energies. This result indicates that formaldehyde is able to dissipate much larger amounts of incident kinetic energy during collision with the $\mathrm{Au}(111)$ surface. The polyatomic prolate top molecule ethane exhibits a similar degree of enhanced trapping in the range $E_{\mathrm{i}}=0.05-0.4 \mathrm{eV}$.

In classical trajectory simulations of ethane trapping on $\mathrm{Pt}(111)$ reported in ref. 4 , the excitation of molecular rotation was found to play a decisive role in the trapping probability, especially for the highest normal incidence kinetic energy investigated $(0.4 \mathrm{eV})$. In trajectories where the first bounce led to sufficient excitation of molecular rotation, the molecule became temporarily "rotationally trapped" in the surface potential. The average energy transferred to phonons during the first bounce was the similar in the "rotational trapping" and "single-bounce scattering" channels. In $>90 \%$ of "rotationally trapped" trajectories, the initially stored rotational energy was dissipated to surface degrees of freedom during subsequent bounces, leading eventually to a relaxed trapping configuration. Thus, the degree of rotational excitation that occurred during the first bounce determined the eventual fate of the molecule (trapping or direct scattering) to within $10 \%$ uncertainty. In ref. 30 , it was remarked that a similar mechanism might explain the trapping of formaldehyde on $\mathrm{Au}(111)$ at $E_{\mathrm{i}}=0.58 \mathrm{eV}$.

We examine the feasibility of the "rotational trapping" mechanism by analysis of our experimentally measured rotational distribution. Assuming that the average phonon excitation during the first bounce is determined by a hard sphere collision model involving a single gold atom, a 30 amu formaldehyde molecule will lose $46 \%$ of its incident energy to phonon excitation. ${ }^{30}$ At $E_{\mathrm{i}}=0.58 \mathrm{eV}$, an additional $0.31 \mathrm{eV}$ of kinetic energy must therefore, on average, be dissipated in order for trapping to occur. If we make the (admittedly naive) 

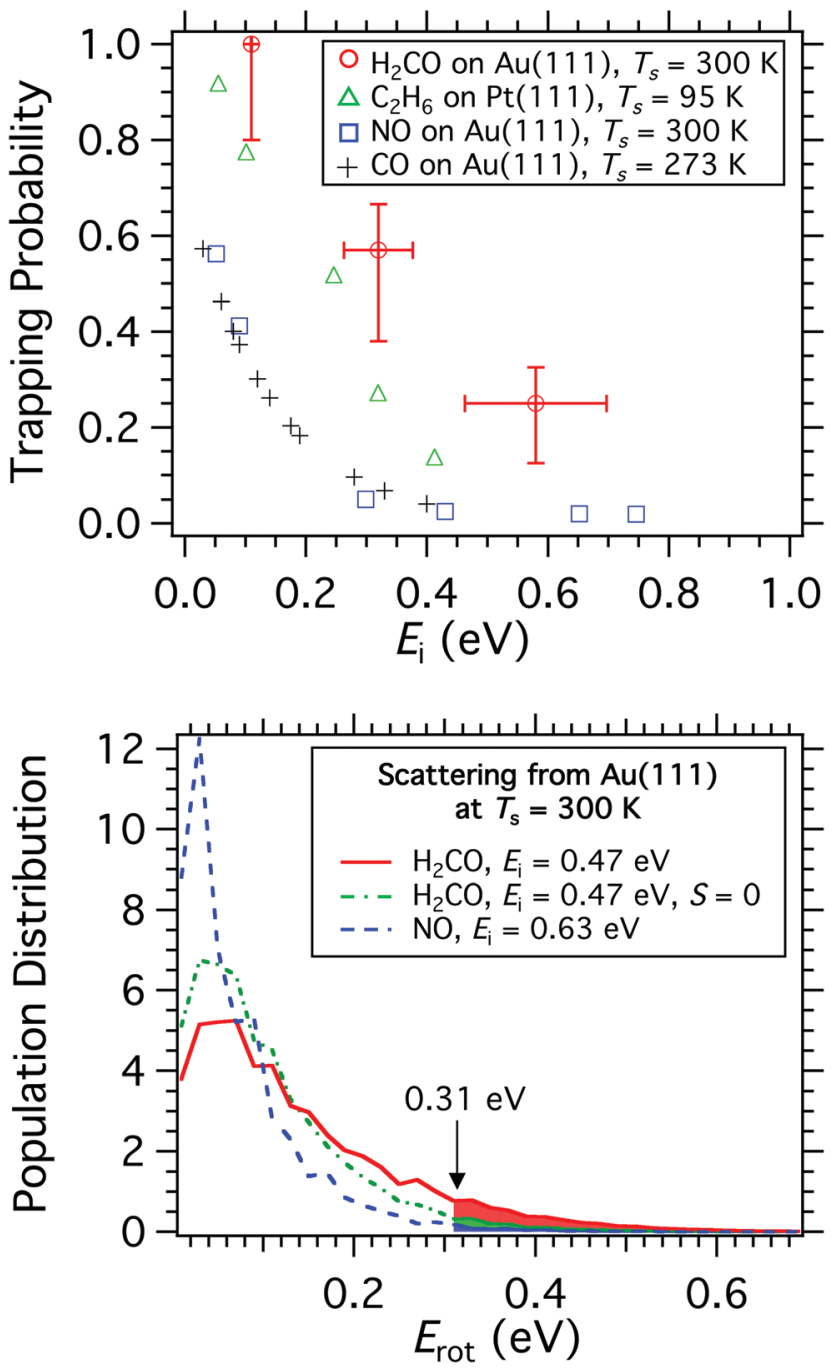

Fig. 8 In the top panel, the estimated trapping probability of formaldehyde on $\mathrm{Au}(111)^{30}$ is plotted as a function of incidence kinetic energy. Measured trapping probabilities from three other weakly bound molecular scattering systems with similar molecular and surface atom masses are shown for comparison. Data for ethane on $\mathrm{Pt}(111)$ ( $0.33 \mathrm{eV}$ binding energy) is taken from ref. 46; $\mathrm{NO}$ on $\mathrm{Au}(111)(0.24 \mathrm{eV}$ binding energy) is from ref. 47; and $\mathrm{CO}$ on $\mathrm{Au}(111)\left(0.18 \mathrm{eV}\right.$ binding energy $\left.{ }^{48}\right)$ is from ref. 49 . The bottom panel shows the normalized rotational energy distribution that results from direct scatter of formaldehyde at $E_{\mathrm{i}}=0.47 \mathrm{eV}$. Shown for comparison are the distribution that is obtained when the $S$ parameter, describing the contribution of the $a$-axis rotational rainbow, is set to zero. Also shown is the distribution that is obtained from the direct scatter of NO from $\mathrm{Au}(111)$ at $E_{\mathrm{i}}=0.63 \mathrm{eV}$, reported in ref. 45 . The tails of the distribution with $E_{\text {rot }} \geq 0.31 \mathrm{eV}$ are shaded. This is the portion of the distribution with sufficient rotational excitation to give rise to rotationally mediated trapping, according to a simple model (see text).

assumption that there is no correlation between energy transfer to phonons and to molecular rotation, then the trapping probability that will result from rotational excitation mechanisms will be given approximately by the fraction of molecules with $\geq 0.31 \mathrm{eV}$ of rotational excitation. In our fitted rotational distribution, shown in the bottom panel of Fig. $8,9.8 \%$ of directly scattered formaldehyde molecules at $E_{\mathrm{i}}=0.47 \mathrm{eV}$ are excited to this energy. This is less than the observed trapping probability of $25 \%$ (at $E_{\mathrm{i}}=0.58 \mathrm{eV}$ ), but is obtained at a slightly lower value of $E_{\mathrm{i}}$ and represents only the rotational energy distribution in the direct scatter channel (i.e., in molecules that do not trap). The semi-quantitative agreement is therefore a strong argument that rotation plays a major role in the trapping mechanism. On the other hand, in the ground state rotational distribution of NO molecules scattered from $\mathrm{Au}(111)$ at $E_{\mathrm{i}}=0.60 \mathrm{eV}$ (shown for comparison in Fig. 8) only $1.1 \%$ of molecules are excited to $E_{\text {rot }} \geq 0.31 \mathrm{eV} .^{45}$ This is consistent with the observation that the trapping probability in the NO on $\mathrm{Au}(111)$ system is an order of magnitude less than that of formaldehyde. Finally, we note that without the $a$-axis rainbow (i.e., with the $S$ parameter of eqn (1) set to zero) the fraction of directly scattered formaldehyde molecules with $\geq 0.31 \mathrm{eV}$ of rotational excitation is only $3.1 \%$ at $E_{\mathrm{i}}=0.47 \mathrm{eV}$. (This distribution is also shown for comparison in Fig. 8) This underscores the importance of $a$-axis rotation to the high-energy tail of the rotational distribution and points to the likely contribution of this additional degree of rotational freedom (not present in diatomic molecules) to trapping at higher incidence kinetic energies.

\section{Conclusions}

In this work, we have used a $1+1^{\prime}$ REMPI scheme to characterize the ground state rotational distribution that results from molecular beam surface scattering of formaldehyde from $\mathrm{Au}(111)$ in both the trapping/desorption and direct scatter regime. Our work is the first demonstration of rotational distribution measurements from surface scattering of an asymmetric top molecule. In the direct scatter channel, there is a pronounced propensity to excite a high degree of $a$-axis (twirling) rotation, consistent with a high- $J_{a}$ axis-specific rotational rainbow. Classical trajectory simulations allow the result to be interpreted as a steric effect, arising from excitation to high $J_{a}$ over a wide range of incident orientation space over which $\partial J_{a} / \partial \psi_{\mathrm{i}}=0$. We note that $a$-axis rotation makes a significant contribution to the high-energy tail of the rotational distribution and may therefore play an important role in the trapping mechanism at high incidence energy. Since the rotational rainbow arises primarily from steric effects and inertial considerations, one can expect such effects to occur quite generally in the scattering of weakly bound, small prolate (or nearprolate) top molecules in which the rotation of light, off-axis hydrogen atoms is readily excited.

\section{Acknowledgements}

The authors thank Dirk Schwarzer for building the nozzle used in this experiment and for providing continuing support. G. B. P. thanks Russ Cooper for providing raw data from ref. 45 . A. M. W. and G. B. P. acknowledge support from the Alexander von Humboldt Foundation. T. S. gratefully acknowledges financial support by the Deutsche Forschungsgemeinschaft under grant SCHA 1946/2-1. Open Access funding provided by the Max Planck Society. 


\section{References}

1 C. T. Rettner, C. B. Mullins, D. S. Bethune, D. J. Auerbach, E. K. Schweizer and W. H. Weinberg, Molecular beam studies of trapping dynamics, J. Vac. Sci. Technol., A, 1990, 8(3), 2699-2704.

2 M. A. Hines and R. N. Zare, The interaction of CO with Ni(111): rainbows and rotational trapping, J. Chem. Phys., 1993, 98(11), 9134-9147.

3 D. Kulginov, M. Persson, C. Åkerlund, I. Zorić and B. Kasemo, $\mathrm{CO}_{2}$ sticking on $\mathrm{Pt}(111)$ : the role of kinetic energy and internal degrees of freedom, J. Vac. Sci. Technol., A, 1995, 13(3), 1511-1516.

4 J. A. Stinnett, R. J. Madix and J. C. Tully, Stochastic simulations of the trapping of ethane on $\mathrm{Pt}(111)$ from a realistic potential: the roles of energy transfer processes and surface corrugation, J. Chem. Phys., 1996, 104(8), 3134-3142.

5 J. F. Weaver and R. J. Madix, Trapping dynamics of isobutane, $n$-butane, and neopentane on $\operatorname{Pt}(111)$ : effects of molecular weight and structure, J. Chem. Phys., 1999, 110(21), 10585-10598.

6 R. Schlögl, Heterogeneous catalysis, Angew. Chem., Int. Ed., 2015, 54(11), 3465-3520.

7 F. Zaera, Probing catalytic reactions at surfaces, Prog. Surf. Sci., 2001, 69(1-3), 1-98.

8 R. R. Cavanagh and D. S. King, Rotational- and spin-state distributions: NO thermally desorbed from $\mathrm{Ru}(001)$, Phys. Rev. Lett., 1981, 47, 1829-1832.

9 M. C. Lin and G. Ertl, Laser probing of molecules desorbing and scattering from solid surfaces, Annu. Rev. Phys. Chem., 1986, 37, 587-615.

10 J. Kimman, C. T. Rettner, D. J. Auerbach, J. A. Barker and J. C. Tully, Correlation between kinetic-energy transfer to rotation and to phonons in gas-surface collisions of NO with Ag(111), Phys. Rev. Lett., 1986, 57, 2053-2056.

11 L. V. Novakoski and G. M. McClelland, Orientation of $\mathrm{CHF}_{3}$ desorbed and scattered from $\mathrm{Ag}(111)$ : measurements using electrostatic focusing, Phys. Rev. Lett., 1987, 59, 1259-1262.

12 L. Vattuone, L. Savio, F. Pirani, D. Cappelletti, M. Okada and M. Rocca, Interaction of rotationally aligned and of oriented molecules in gas phase and at surfaces, Prog. Surf. Sci., 2010, 85(1-4), 92-160.

13 M. G. Tenner, F. H. Geuzebroek, E. W. Kuipers, A. E. Wiskerke, A. W. Kleyn, S. Stolte and A. Namiki, Orientation dependence of rotational excitation in NO scattering from $\mathrm{Ag}(111)$, Chem. Phys. Lett., 1990, 168(1), 45-50.

14 F. H. Geuzebroek, A. E. Wiskerke, M. G. Tenner, A. W. Kleyn, S. Stolte and A. Namiki, Rotational excitation of oriented molecules as a probe of molecule-surface interaction, J. Phys. Chem., 1991, 95(21), 8409-8421.

15 A. W. Kleyn, Rotational rainbow scattering of oriented molecules, Surf. Rev. Lett., 1994, 1(1), 157-173.

16 N. Bartels, K. Golibrzuch, C. Bartels, L. Chen, D. J. Auerbach, A. M. Wodtke and T. Schäfer, Dynamical steering in an electron transfer surface reaction: oriented $\mathrm{NO}\left(v=3,0.08<E_{\mathrm{i}}<0.89 \mathrm{eV}\right)$ relaxation in collisions with a $\mathrm{Au}(111)$ surface, J. Chem. Phys., 2014, 140(5), 054710.
17 A. W. Kleyn and T. C. M. Horn, Rainbow scattering from solid surfaces, Phys. Rep., 1991, 199(4), 191-230.

18 A. W. Kleyn, A. C. Luntz and D. J. Auerbach, Rotational energy transfer in direct inelastic surface scattering: NO on Ag(111), Phys. Rev. Lett., 1981, 47, 1169-1172.

19 R. Schinke, Rotational rainbows in diatom(solid) surface scattering, J. Chem. Phys., 1982, 76(5), 2352-2359.

$20 \mathrm{H}$. Voges and R. Schinke, On the dynamics of molecule/ surface scattering, Chem. Phys. Lett., 1983, 95(3), 221-225.

21 C. W. Muhlhausen, L. R. Williams and John C. Tully, Dynamics of gas-surface interactions: scattering and desorption of NO from $\mathrm{Ag}(111)$ and $\mathrm{Pt}(111), \mathrm{J}$. Chem. Phys., 1985, 83(5), 2594-2606.

22 J. A. Barker, A. W. Kleyn and D. J. Auerbach, Rotational energy distributions in molecule surface scattering: model calculations for NO/Ag(111), Chem. Phys. Lett., 1983, 97(1), 9-13.

23 J. C. Polanyi and R. J. Wolf, Dynamics of simple gas-surface interaction. II. Rotationally inelastic collisions at rigid and moving surfaces, J. Chem. Phys., 1985, 82(3), 1555-1566.

24 D. C. Jacobs and R. N. Zare, Simplified trajectory method for modeling gas-surface scattering: the NO/Pt(111) system, J. Chem. Phys., 1989, 91(5), 3196-3207.

25 M. E. Coltrin and B. D. Kay, Quasiclassical trajectory study of rotational energy transfer in the scattering of $\mathrm{NH}_{3}$ from a flat, rigid gold surface, J. Chem. Phys., 1988, 89(1), 551-561.

26 B. G. Perkins, Jr. and D. J. Nesbitt, Quantum-state-resolved $\mathrm{CO}_{2}$ scattering dynamics at the gas-liquid interface: incident collision energy and liquid dependence, J. Phys. Chem. B, 2006, 110(34), 17126-17137.

27 K. Golibrzuch, J. H. Baraban, P. R. Schirhatti, J. Werdecker, C. Bartels and A. M. Wodtke, Observation of translation-tovibration excitation in acetylene scattering from $\mathrm{Au}(111)$ : a REMPI based approach, Z. Phys. Chem., 2015, 229(10-12), 1929-1949.

28 A. C. Wight and R. E. Miller, Rainbow scattering of methane from $\operatorname{LiF}(100)$ : probing the corrugation and anisotropy of the gas-surface potential, J. Chem. Phys., 1998, 109(5), 1976-1982.

29 B. D. Kay, T. D. Raymond and M. E. Coltrin, Observation of a dynamical propensity rule in rotationally inelastic gassurface scattering: $\mathrm{NH}_{3}$ on $\mathrm{Au}(111)$, Phys. Rev. B: Condens. Matter Mater. Phys., 1987, 36, 6695-6697.

30 B. C. Krüger, G. Barratt Park, S. Meyer, R. J. V. Wagner, A. M. Wodtke and T. Schäfer, Trapping-desorption and direct-scattering of formaldehyde at Au(111), Phys. Chem. Chem. Phys., 2017, DOI: 10.1039/c7cp03907g.

31 G. Barratt Park, B. C. Krüger, S. Meyer, D. Schwarzer and T. Schäfer, The $\nu_{6}$ fundamental frequency of the $\tilde{A}$ state of formaldehyde and Coriolis perturbations in the $3 \nu_{4}$ level, J. Chem. Phys., 2016, 144(194308).

32 G. Barratt Park, B. C. Krüger, S. Meyer, A. M. Wodtke and T. Schäfer, A $1+1^{\prime}$ resonance-enhanced multiphoton ionization scheme for rotationally state-selective detection of formaldehyde via the $\tilde{\mathrm{A}}{ }^{1} \mathrm{~A}_{2} \leftarrow \tilde{\mathrm{X}}{ }^{1} \mathrm{~A}^{1}$ transition, Phys. Chem. Chem. Phys., 2016, 18, 22355-22363. 
33 D. J. Clouthier and D. A. Ramsay, The spectroscopy of formaldehyde and thioformaldehyde, Annu. Rev. Phys. Chem., 1983, 34, 31-58.

34 H. S. P. Müller, G. Winnewisser, J. Demaison, A. Perrin and A. Valentin, The ground state spectroscopic constants of formaldehyde, J. Mol. Spectrosc., 2000, 200(1), 143-144.

35 E. W. Kuipers, M. G. Tenner, A. W. Kleyn and S. Stolte, Observation of steric effects in gas-surface scattering, Nature, 1988, 334(6181), 420-422.

36 J. C. Tully and M. J. Cardillo, Dynamics of molecular motion at single-crystal surfaces, Science, 1984, 223(4635), 445-450.

37 D. C. Burleigh, A. B. McCoy and E. L. Sibert, An accurate quartic force field for formaldehyde, J. Chem. Phys., 1996, 104(2), 480-487.

38 A. K. Rappe, C. J. Casewit, K. S. Colwell, W. A. Goddard III and W. M. Skiff, UFF, a full periodic table force field for molecular mechanics and molecular dynamics simulations, J. Am. Chem. Soc., 1992, 114(25), 10024-10035.

39 W.-K. Chen, S.-H. Liu, M.-J. Cao, Q.-G. Yan and C.-H. Lu, Adsorption and dissociation of methanol on $\mathrm{Au}(111)$ surface: a first-principles periodic density functional study, J. Mol. Struct.: THEOCHEM, 2006, 770(1-3), 87-91.

40 S. Liu, P. Jin, D. Zhang, C. Hao and X. Yang, Reaction mechanism for methanol oxidation on $\mathrm{Au}(111)$ : a density functional theory study, Appl. Surf. Sci., 2013, 265, 443-451.

41 L. Wang, C. He, W. Zhang, Z. Li and J. Yang, Methanolselective oxidation pathways on Au surfaces: a first-principles study, J. Phys. Chem. C, 2014, 118(31), 17511-17520.
42 R. M. Logan and J. C. Keck, Classical theory for the interaction of gas atoms with solid surfaces, J. Chem. Phys., 1968, 49(2), 860-876.

43 E. W. Kuipers, M. G. Tenner, M. E. M. Spruit and A. W. Kleyn, Differential trapping probabilities and desorption of physisorbed molecules: application to NO/Ag(111), Surf. Sci., 1988, 205(1-2), 241-268.

44 C. R. Arumainayagam, M. C. McMaster, G. R. Schoofs and R. J. Madix, Dynamics of molecular $\mathrm{CH}_{4}$ adsorption on Pt(111), Surf. Sci., 1989, 222(1), 213-246.

45 R. Cooper, Z. Li, K. Golibrzuch, C. Bartels, I. Rahinov, D. J. Auerbach and A. M. Wodtke, On the determination of absolute vibrational excitation probabilities in moleculesurface scattering: case study of NO on Au(111), J. Chem. Phys., 2012, 137(6), 064705.

46 C. R. Arumainayagam, G. R. Schoofs, M. C. McMaster and R. J. Madix, Dynamics of molecular adsorption of ethane with platinum(111): a supersonic molecular beam study, J. Phys. Chem., 1991, 95(3), 1041-1047.

47 A. M. Wodtke, H. Yuhui and D. J. Auerbach, Insensitivity of trapping at surfaces to molecular vibration, Chem. Phys. Lett., 2005, 413(4-6), 326-330.

48 D. P. Engelhart, R. J. V. Wagner, A. Meling, A. M. Wodtke and T. Schäfer, Temperature programmed desorption of weakly bound adsorbates on $\mathrm{Au}(111)$, Surf. Sci., 2016, 650, 11-16.

49 C. T. Rettner, The search for direct vibrational excitation in gas-surface collisions of $\mathrm{CO}$ with $\mathrm{Au}(111), \mathrm{J}$. Chem. Phys., 1993, 99(7), 5481-5489. 